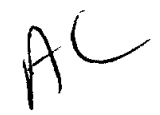

fTur $93-48$

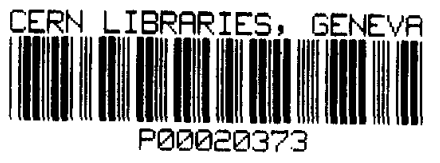

$8 x+3405$

\title{
Semileptonic decays of baryons containing two heavy quarks *
}

\author{
Miguel Angel Sanchis-Lozano \\ Departamento de Física Teórica \\ and \\ Instituto de Física Corpuscular (IFIC) \\ Centro Mixto Universidad de Valencia-CSIC \\ Dr. Moliner 50, E-46100 Burjassot, Valencia (Spain)
}

November 22, 1993

\begin{abstract}
Semileptonic weak decays of doubly heavy baryons are analyzed and compared to the analogous decays of heavy quarkonia, showing similar underlying dynamics at low recoil.
\end{abstract}

FTUV: 93-48

IFIC: $93-28$

e-mail : mas@evalvx.ific.uv.es

* Research partially supported by CICYT under contract FEN 93-0234 and by IVEI 

The standard application of the heavy quark effective theory (HQET) is to deal with hadronic systems containing a single heavy quark [1]. However, the possibility of extending this formalism to hadrons with two heavy quarks was soon considered by Savage and Wise [2], suggesting that a baryon composed of two heavy quarks and one light quark should look like a heavy-light "meson". More recently several authors have applied HQET to heavyheavy hadrons like $B_{c}$ mesons [3] or $J / \psi$ and $\Upsilon$ resonances [4] [5]. However, some caution must be taken in defining the heavy quark effective QCD Lagrangian for systems containing two heavy quarks since the $O\left(1 / m_{Q}\right)$ kinetic term cannot be neglected even in the $m_{Q} \rightarrow \infty$ limit, as emphasized in the above-quoted references. Such term containing the heavy quark mass explicitly breaks the flavor symmetry though leaves the spin symmetry as a useful approximation in the preasymptotic quark mass region.

In baryons with two heavy quarks $(Q Q q)$, the massive quark pair bind into a $\overline{3}$ source of color with radius $\simeq 1 / \alpha_{s}\left(m_{Q}\right) m_{Q}$, interacting with the light degrees of freedom as a static antiquark [2] [6]. This description makes sense if the size of the "heavy core" is small compared to the typical length of the hadron $1 / \Lambda_{Q C D}$. Even so, the finite masses of bottom and especially charm quarks would yield important corrections to the pointlike character of the diquark, also due in part to the fact that the $(Q Q)$ Coulomb-like potential is half as attractive as the $(Q \bar{Q})$ potential. Hence, our later estimates should be considered as a starting point of a more rigorous treatment for constituent charm and even bottom quarks.

If the baryon contains two heavy quarks of different flavors $a$ and $b$, there are two possible spin configurations of the compact diquark at lowest level. If the $\left(Q_{a} Q_{b}\right)$ form a spinsinglet state, the ground-state hadron will have spin $1 / 2$ which we shall refer to as a $\Lambda_{a b}$ baryon. If the $\left(Q_{a} Q_{b}\right)$ form a spin-triplet state, the ground-state hadron can be either a spin-1/2 baryon (denoted by $\Sigma_{a b}$ ) or a spin-3/2 baryon (denoted by $\Sigma_{a b}^{*}$ ). Heavy quark spin 
symmetry implies the mass degeneracy among all these hadronic states [2] [7]. Actually, the chromo-magnetic hyperfine interaction should resolve the degeneracy leading to the mass level: $m\left(\Sigma_{a b}\right)<m\left(\Lambda_{a b}\right)<m\left(\Sigma_{a b}^{*}\right)$, according to [6].

If the heavy diquark in the baryon undergoes a semileptonic weak decay ${ }^{1}$ yielding another heavy diquark, the light constituent should play only a marginal role at low recoil, in a similar way to transitions between ordinary heavy-light mesons. Therefore, the overlap between the light degrees of freedom of the initial and final doubly heavy baryons could be described in terms of the Isgur-Wise function as well. The presence of the light component should not change in principle the overall decay rate of the heavy diquark near zero recoil but, however, would channel the decay into different spin-states of the final baryon according to some selection rules. This suggests to perform a detailed comparison between the semileptonic decay of another heavy-heavy bound system like quarkonium examined in ref. [5] and the results obtained by White and Savage for doubly heavy baryons in [7].

Let us start by writing their expressions of the matrix elements for transitions of a baryon $Q_{a} Q_{b} q$ of mass $m_{1}$ with four-velocity $v_{1}$ into another baryon $Q_{a} Q_{c} q$ of mass $m_{2}$ with fourvelocity $v_{2}$ induced by the weak current $O_{c b}^{\mu}=\bar{Q}_{c} \gamma^{\mu}\left(1-\gamma_{5}\right) Q_{b}$,

$$
\begin{aligned}
\left\langle\Lambda_{a c}\left(v_{2}\right)\left|O_{c b}^{\mu}\right| \Lambda_{a b}\left(v_{1}\right)\right\rangle= & \frac{\eta_{a b c}\left(v_{1}, v_{2}\right)}{4 \tilde{m}}\left(v_{1}+v_{2}\right)^{\mu} \bar{u}_{2}\left(v_{2}\right) u_{1}\left(v_{1}\right) \\
\left\langle\Lambda_{a c}\left(v_{2}\right)\left|O_{c b}^{\mu}\right| \Sigma_{a b}\left(v_{1}\right)\right\rangle= & \frac{i \eta_{a b c}\left(v_{1}, v_{2}\right)}{2 \sqrt{3} \tilde{m}} \bar{u}_{2}\left(v_{2}\right)\left(v_{1}^{\mu}+\gamma^{\mu}\right) \gamma_{5} u_{1}\left(v_{1}\right) \\
\left\langle\Lambda_{a c}\left(v_{2}\right)\left|O_{c b}^{\mu}\right| \Sigma_{a b}^{*}\left(v_{1}\right)\right\rangle= & \frac{\eta_{a b c}\left(v_{1}, v_{2}\right)}{2 \tilde{m}} \bar{u}_{2}\left(v_{2}\right) \psi_{1}^{\mu}\left(v_{1}\right) \\
\left\langle\Sigma_{a c}\left(v_{2}\right)\left|O_{c b}^{\mu}\right| \Sigma_{a b}\left(v_{1}\right)\right\rangle= & \frac{\eta_{a b c}\left(v_{1}, v_{2}\right)}{6 \tilde{m}} \bar{u}_{2}\left(v_{2}\right)\left[\frac{1}{2}\left(v_{1}+v_{2}\right)^{\mu}\left(2+v_{1} \cdot v_{2}\right)+\left(v_{1}-v_{2}\right)^{\mu} \gamma_{5}+\right. \\
& \left.+2 \gamma^{\mu} \gamma_{5}+i \epsilon^{\mu \alpha \beta \sigma} \gamma_{\alpha} v_{1 \beta} v_{2 \sigma}\right] u_{1}\left(v_{1}\right)
\end{aligned}
$$

\footnotetext{
${ }^{1}$ Under the assumption that strong decay cannot take place according to the theoretical mass expectations for baryons containing two heavy (charm and/or bottom) quarks [6] [8]. Still, radiative decays might exist through $M_{1}$ transitions between some of those baryon states.
} 


$$
\begin{aligned}
<\Sigma_{a c}\left(v_{2}\right)\left|O_{c b}^{\mu}\right| \Sigma_{a b}^{*}\left(v_{1}\right)>= & \frac{-i \eta_{a b c}\left(v_{1}, v_{2}\right)}{4 \sqrt{3} \tilde{m}} \bar{u}_{2}\left(v_{2}\right)\left[\left(v_{1}+v_{2}\right)^{\mu} v_{2}^{\alpha}+\right. \\
& \left.+i \epsilon^{\mu \alpha \beta \sigma}\left(v_{2 \beta}-\gamma_{\beta}\right)\left(v_{1}+v_{2}\right)_{\sigma}\right] \gamma_{5} \psi_{1 \alpha}\left(v_{1}\right) \\
<\Sigma_{a c}^{*}\left(v_{2}\right)\left|O_{c b}^{\mu}\right| \Sigma_{a b}^{*}\left(v_{1}\right)>= & \frac{\eta_{a b c}\left(v_{1}, v_{2}\right)}{4 \tilde{m}} \bar{\psi}_{2 \alpha}\left(v_{2}\right)\left[-g^{\alpha \beta}\left(v_{1}+v_{2}\right)^{\mu}+\right. \\
& \left.+i \epsilon^{\mu \alpha \beta \sigma}\left(v_{1}+v_{2}\right)_{\sigma}\right] \psi_{1 \beta}\left(v_{1}\right)
\end{aligned}
$$

where $\tilde{m}=\sqrt{m_{1} m_{2}} ; u_{\Lambda}, u_{\Sigma}$ are the spinor wave functions for $\Lambda, \Sigma$ normalized to $\bar{u}_{i} u_{i}=2 m_{i}$ satisfying $\psi_{i}^{\prime} u_{i}=u_{i} ; \psi_{\Sigma}^{\mu}$. is the Rarita-Schwinger wave function of the $\Sigma^{*}$ normalized to $\psi_{i}^{\mu} \psi_{i \mu}=-2 m_{i}$ satisfying $\psi_{i} \psi_{i}^{\mu}=\psi_{i}^{\mu}, v_{i \mu} \psi_{i}^{\mu}=\gamma_{\mu} \psi_{i}^{\mu}=0$. Let us also recall that the $\Lambda_{a b}$ ground-state can only exist for unequal heavy flavors $a \neq b$.

The form factor $\eta_{a b c}\left(v_{1}, v_{2}\right)$ will be factorized as the product of the Isgur-Wise function $\xi\left(v_{1} \cdot v_{2}\right)$ concerning the light degrees of freedom, and the wavefunction overlap $\hat{\eta}_{a b c}\left(v_{1}, v_{2}\right)$ between the initial and final heavy diquark systems, namely

$$
\eta_{a b c}\left(v_{1}, v_{2}\right)=\xi\left(v_{1} \cdot v_{2}\right) \hat{\eta}_{a b c}\left(v_{1}, v_{2}\right)
$$

In the special case $a=b$ let us note that $\hat{\eta}_{b b c}\left(v_{1}, v_{2}\right)$ is the analog of the corresponding overlap function defined in ref. [5] for the weak decays of heavy quarkonium (however both form factors should not coincide because of the different binding force and thereby distinct size of the $Q_{i} Q_{j}$ and $Q_{i} \bar{Q}_{j}$ systems).

Since no heavy quark flavor symmetry can be applied to heavy-heavy systems, neither $\hat{\eta}_{a b c}$ nor consequently $\eta_{a b c}$ are normalized to unity at zero recoil but can be calculated in a model-dependent way as in [7] [3] or in [9]. Instead, near this kinematic point $\xi\left(v_{1} \cdot v_{2} \simeq 1\right)$ 
can be approximated to unity. Thereby, at $v_{1}=v_{2}=v$ one gets ${ }^{2}$ from the above expressions

$$
\begin{aligned}
& <\Lambda_{a c}(v)\left|O_{c b}^{\mu}\right| \Lambda_{a b}(v)>\sim \frac{\hat{\eta}_{a b c}}{2 \tilde{m}} v^{\mu} \bar{u}_{2}(v) u_{1}(v) \\
& <\Lambda_{a c}(v)\left|O_{c b}^{\mu}\right| \Sigma_{a b}(v)>\sim \frac{i \hat{\eta}_{a b c}}{2 \sqrt{3} \tilde{m}} \bar{u}_{2}(v) \gamma^{\mu} \gamma_{5} u_{1}(v) \\
& <\Lambda_{a c}(v)\left|O_{c b}^{\mu}\right| \Sigma_{a b}^{*}(v)>\sim \frac{\hat{\eta}_{a b c}}{2 \tilde{m}} \bar{u}_{2}(v) \psi_{1}^{\mu}(v) \\
& <\Sigma_{a c}(v)\left|O_{c b}^{\mu}\right| \Sigma_{a b}(v)>\sim \frac{\hat{\eta}_{a b c}}{6 \tilde{m}} \bar{u}_{2}(v)\left[3 v^{\mu}+2 \gamma^{\mu} \gamma_{5}\right] u_{1}(v) \\
& <\Sigma_{a c}(v)\left|O_{c b}^{\mu}\right| \Sigma_{a b}^{*}(v)>\sim \frac{-i \hat{\eta}_{a b c}}{2 \sqrt{3} \tilde{m}} \bar{u}_{2}(v) \psi_{1}^{\mu}(v) \\
& <\Sigma_{a c}^{*}(v)\left|O_{c b}^{\mu}\right| \Sigma_{a b}^{*}(v)>\sim \frac{\hat{\eta}_{a b c}}{2 \tilde{m}} \bar{\psi}_{2 \alpha}(v)\left[-g^{\alpha \beta} v^{\mu}+i \epsilon^{\mu \alpha \beta \sigma} v_{\sigma}\right] \psi_{1 \beta}(v)
\end{aligned}
$$

Next, we form the hadronic tensors at zero recoil from the currents (8-13) averaging and summing over initial and final polarizations respectively ${ }^{3}$. For an initial state $\Lambda_{a b}$ we get aside a common multiplicative $\hat{\eta}_{a b c}^{2}\left(v_{1}=v_{2}\right)$ throughout

$$
\begin{aligned}
T^{\mu \nu}\left[\Lambda_{a b} \rightarrow \Lambda_{a c}\right] & \sim v^{\mu} v^{\nu} \\
T^{\mu \nu}\left[\Lambda_{a b} \rightarrow \Sigma_{a c}\right] \simeq \frac{1}{2} T^{\mu \nu}\left[\Lambda_{a b} \rightarrow \Sigma_{a c}^{*}\right] & \sim \frac{1}{3}\left(-g^{\mu \nu}+v^{\mu} v^{\nu}\right)
\end{aligned}
$$

If the initial state is a $\Sigma_{a b}$

$$
\begin{aligned}
& T^{\mu \nu}\left[\Sigma_{a b} \rightarrow \Lambda_{a c}\right] \sim \frac{1}{3}\left(-g^{\mu \nu}+v^{\mu} v^{\nu}\right) \\
& T^{\mu \nu}\left[\Sigma_{a b} \rightarrow \Sigma_{a c}\right] \sim \frac{4}{9}\left(-g^{\mu \nu}+v^{\mu} v^{\nu}\right)+v^{\mu} v^{\nu}
\end{aligned}
$$

\footnotetext{
${ }^{2}$ Let us remark that the amplitude for the transition $\Sigma_{a b}^{*} \rightarrow \Sigma_{a c}$ does not vanish at zero recoil contrary to the result found in [7] (see [10]). In fact, this is readily seen from equation (5) by using the identity $i \epsilon^{\mu \alpha \beta \sigma} \gamma_{\beta} \gamma_{5}=$ $\gamma^{\mu} \gamma^{\alpha} \gamma^{\sigma}-g^{\mu \alpha} \gamma^{\sigma}-g^{\alpha \sigma} \gamma^{\mu}+g^{\mu \sigma} \gamma^{\alpha}$. Upon contraction with $v_{\sigma} \psi_{1 \alpha}(v)$, the first, third and last terms in the rhs vanish in virtue of the usual properties of the Rarita-Schwinger spinor, but the second one yields equation (12).

${ }^{3}$ For the spin- $3 / 2$ states we have used:

$$
\frac{1}{2 m_{i}} \sum_{4 p o l} \psi_{i}^{\mu} \bar{\psi}_{i}^{\nu}=\left(\frac{1+\psi}{2}\right)\left[-g^{\mu \nu}+\frac{1}{3} \gamma^{\mu} \gamma^{\nu}+\frac{2}{3} v^{\mu} v^{\nu}-\frac{1}{3}\left(v^{\mu} \gamma^{\nu}-v^{\nu} \gamma^{\mu}\right)\right]
$$
}




$$
T^{\mu \nu}\left[\Sigma_{a b} \rightarrow \Sigma_{a c}^{*}\right] \sim \frac{2}{9}\left(-g^{\mu \nu}+v^{\mu} v^{\nu}\right)
$$

and for the $\Sigma_{a b}^{*}$ initial state

$$
\begin{aligned}
& T^{\mu \nu}\left[\Sigma_{a b}^{*} \rightarrow \Lambda_{a c}\right] \sim \frac{1}{3}\left(-g^{\mu \nu}+v^{\mu} v^{\nu}\right) \\
& T^{\mu \nu}\left[\Sigma_{a b}^{*} \rightarrow \Sigma_{a c}\right] \sim \frac{1}{9}\left(-g^{\mu \nu}+v^{\mu} v^{\nu}\right) \\
& T^{\mu \nu}\left[\Sigma_{a b}^{*} \rightarrow \Sigma_{a c}^{*}\right] \sim \frac{5}{9}\left(-g^{\mu \nu}+v^{\mu} v^{\nu}\right)+v^{\mu} v^{\nu}
\end{aligned}
$$

Gathering those transitions giving a final $\Lambda$ baryon one readily finds

$$
\begin{aligned}
T^{\mu \nu}\left[\Lambda_{a b} \rightarrow \Lambda_{a c}\right] & \sim v^{\mu} v^{\nu} \\
T^{\mu \nu}\left[\Sigma_{a b} \rightarrow \Lambda_{a c}\right] & \simeq T^{\mu \nu}\left[\Sigma_{a b}^{*} \rightarrow \Lambda_{a c}\right] \\
& \sim \frac{1}{3}\left(-g^{\mu \nu}+v^{\mu} v^{\nu}\right)
\end{aligned}
$$

whereas for a final $\Sigma$-type baryon (i.e. summing over $\Sigma_{a c}$ and $\Sigma_{a c}^{*}$ modes)

$$
\begin{aligned}
T^{\mu \nu}\left[\Lambda_{a b} \rightarrow \Sigma_{a c} \text { or } \Sigma_{a c}^{*}\right] & \sim-g^{\mu \nu}+v^{\mu} v^{\nu} \\
T^{\mu \nu}\left[\Sigma_{a b} \rightarrow \Sigma_{a c} \text { or } \Sigma_{a c}^{*}\right] & \simeq T^{\mu \nu}\left[\Sigma_{a b}^{*} \rightarrow \Sigma_{a c} \text { or } \Sigma_{a c}^{*}\right] \\
& \sim \frac{2}{3}\left(-g^{\mu \nu}+v^{\mu} v^{\nu}\right)+v^{\mu} v^{\nu}
\end{aligned}
$$

The set of equations (22-25) constitute our main result pointing out the same underlying decay dynamics near zero recoil of the compact diquark in the baryon as heavy quarkonium, in accordance with our previous intuitive considerations. Let us examine this point in more detail.

Eqs. (23), (25) correspond to $1^{+} \rightarrow 0^{+}, 1^{+} \rightarrow 1^{+}$diquark parity-conserving transitions. In a prior work [5], we analized the weak decay of vector resonances like $J / \psi$ or $\Upsilon$, in particular the $\left(Q_{b} \bar{Q}_{b}\right)_{1-} \rightarrow\left(Q_{c} \bar{Q}_{b}\right)_{0-}$ transitions induced by the axial-vector current, and the $\left(Q_{b} \bar{Q}_{b}\right)_{1-} \rightarrow\left(Q_{c} \bar{Q}_{b}\right)_{1-}$ transitions induced by both the vector and axial-vector currents. It 
turns out that the hadronic tensors for both processes at zero recoil are formally identical, disregarding the difference between the $\hat{\eta}$ form factors and a trivial overall $4 m_{1} m_{2}$ factor due to a different normalization of the hadronic states (see the last ref. in [5]). On the other hand, eqs. (22), (24) correspond to the $0^{+} \rightarrow 0^{+}, 0^{+} \rightarrow 1^{+}$diquark transitions. It is straightforward to extend the analogy to the decays of pseudoscalar mesons like $\eta_{c}$, $\eta_{b}$ with $\left(Q_{b} \bar{Q}_{b}\right)_{0^{-}} \rightarrow\left(Q_{c} \bar{Q}_{b}\right)_{0^{-}}$occurring now via the vector current and $\left(Q_{b} \bar{Q}_{b}\right)_{0^{-}} \rightarrow\left(Q_{c} \bar{Q}_{b}\right)_{1}$ - via the axial-vector current, as in transitions between heavy-light mesons [11] (the hadronic tensors being again formally identical at zero recoil).

To make more apparent this similarity we shall apply below the non-recoil approximation to the evaluation of rates for the semileptonic decays of doubly heavy baryons : $Q_{a} Q_{b} q \rightarrow Q_{a} Q_{c} q+\ell \nu$, in a parallel way to ref. [5] ${ }^{4}$. By non-recoil approximation we mean that the hadronic matrix elements are obtained at $v_{1}=v_{2}$, though exact kinematic factors as phase space are used in the calculation of decay rates. Besides we neglect lepton masses limiting our discussion to the $\ell=e, \mu$ generations.

Consequently, we contract the above hadronic tensors with the symmetric part of the (zero mass) leptonic tensor, i.e. $L_{\mu \nu}^{s y m}=\frac{8}{3}\left(q_{\mu} q_{\nu}-q^{2} g_{\mu \nu}\right)$ expressed in terms of the fourmomentum transfer $q=p_{1}-p_{2}$ to the leptonic system $\ell$. Integrating over $q^{2}$ between 0 and $\left(m_{1}-m_{2}\right)^{2}$ similar expressions to those for heavy quarkonium are found

$$
\begin{aligned}
\Gamma\left[\Lambda_{a b} \rightarrow \Lambda_{a c}+\ell \nu\right] & \sim \frac{G_{F}^{2} m_{1}^{5}}{192 \pi^{3}} \varphi(x) f_{12}^{2}\left|V_{b c}\right|^{2} \rightarrow \frac{G_{F}^{2}\left(m_{1}-m_{2}\right)^{5}}{60 \pi^{3}} f_{12}^{2}\left|V_{b c}\right|^{2} \\
\Gamma\left[\Sigma_{a b} \rightarrow \Lambda_{a c}+\ell \nu\right] & \simeq \Gamma\left[\Sigma_{a b}^{*} \rightarrow \Lambda_{a c}+\ell \nu\right] \sim \frac{G_{F}^{2} m_{1}^{5}}{576 \pi^{3}}[\varphi(x)+\phi(x)] f_{12}^{2}\left|V_{b c}\right|^{2} \\
& \rightarrow \frac{G_{F}^{2}\left(m_{1}-m_{2}\right)^{5}}{60 \pi^{3}} f_{12}^{2}\left|V_{b c}\right|^{2}
\end{aligned}
$$

\footnotetext{
${ }^{4}$ Notice that the validity of this approximation as an estimate is supported by the fact that $v_{1} \cdot v_{2}$ is restricted to values close to unity. For example, regarding the constituent $s$-quark as a member of heavy quarks the mass values $m_{1} \simeq 3.8 \mathrm{GeV}[6]$ and $m_{2}=2.473 \mathrm{GeV}[12]$ for the $\Xi_{c c}^{*}$ and $\Xi_{c}^{0}$ respectively lead to $\left.v_{1} \cdot v_{2}\right|_{\max } \simeq 1.09$.
} 


$$
\begin{aligned}
\Gamma\left[\Lambda_{a b} \rightarrow \Sigma_{a c} \text { or } \Sigma_{a c}^{*}+\ell \nu\right] & \sim \frac{G_{F}^{2} m_{1}^{5}}{192 \pi^{3}}[\varphi(x)+\phi(x)] f_{12}^{2}\left|V_{b c}\right|^{2} \rightarrow \frac{G_{F}^{2}\left(m_{1}-m_{2}\right)^{5}}{20 \pi^{3}} f_{12}^{2}\left|V_{b c}\right|^{2} \\
\Gamma\left[\Sigma_{a b} \rightarrow \Sigma_{a c} \text { or } \Sigma_{a c}^{*}+\ell \nu\right] & \simeq \Gamma\left[\Sigma_{a b}^{*} \rightarrow \Sigma_{a c} \text { or } \Sigma_{a c}^{*}+\ell \nu\right] \sim \frac{G_{F}^{2} m_{1}^{5}}{576 \pi^{3}}[5 \varphi(x)+2 \phi(x)] f_{12}^{2}\left|V_{b c}\right|^{2} \\
& \rightarrow \frac{G_{F}^{2}\left(m_{1}-m_{2}\right)^{5}}{20 \pi^{3}} f_{12}^{2}\left|V_{b c}\right|^{2}
\end{aligned}
$$

for each massless leptonic generation and degenerate final baryons.

The transition form factor assumed nearly constant over the whole allowed $q^{2}$ range is given by $f_{12}=\sqrt{m_{2} / m_{1}} \hat{\eta}_{a b c}\left(v_{1}=v_{2}\right)$. With respect to the $\varphi$ and $\phi$ phase space factors depending on $x=m_{2} / m_{1}$ their expressions can be found in the last reference of [5]. The rhs limits correspond to the non-relativistic approximation $x \rightarrow 1$ retaining only the leading terms: $\varphi(x) \simeq \phi(x) / 2 \sim 16 / 5(1-x)^{5}$.

In summary, considering the ideal limit of infinitely heavy quarks and the (arguable [4]) assumption of the full validity of the spin symmetry, eqs. (26) and (27) yield a relative branching fraction $r=3$ of modes with a final $\Sigma$-type $\left(\Sigma\right.$ or $\left.\Sigma^{*}\right)$ baryon to modes with a final $\Lambda$-type baryon (for unequal final heavy flavors), no matters that the initial state is a $\Lambda$ - or a $\Sigma$-type baryon. In fact, an equivalent enhancing factor of 3 in favor of final vector hadrons with respect to pseudoscalars is expected as well in semileptonic decays of either heavy-light mesons [13] [11] or heavy-heavy mesons [5] as asymptotic predictions.

In reality, the breaking of the mass degeneracy between different spin-states may imply appreciable effects on rates especially in charmed baryons because of the strong dependence of the phase space factors on the mass difference between the initial and final hadrons. Writing $m\left(\Sigma_{a c}\right)=m\left(\Lambda_{a c}\right)-\frac{3}{4} \delta m$ and $m\left(\Sigma_{a c}^{*}\right)=m\left(\Lambda_{a c}\right)+\frac{1}{4} \delta m[6]$, weighting the contribution from each $\Sigma_{a c}, \Sigma_{a c}^{*}$ mode according to the set of eqs. (14-21), one gets from the non-relativistic expressions in (26), (27) a simple algorithm furnishing roughly $r$ corrected by phase space,

$$
r \simeq 3+k \times \frac{\delta m}{m_{1}-m_{2}\left(\Lambda_{a c}\right)}
$$


where $\delta m$ stands for the hyperfine mass splitting between $\Sigma_{a c}$ and $\Sigma_{a c}^{*} ; k$ takes the values $\simeq 1,8,-2$ for an initial $\Lambda_{a b}, \Sigma_{a b}, \Sigma_{a b}^{*}$ baryon, respectively. In the real world, however, heavy constituent quarks are restricted to charm, beauty and may be also $s$-quark to some extent, whereas top must be left aside as it would not form a bound state. Since $\Lambda_{a b}$ only exists if $a \neq b$, expression (28) only applies to an initial doubly charmed or beautiful $\Sigma$-type baryon. Assuming $\delta m \lesssim 100 \mathrm{MeV}[6][8]$, the correction due to phase space is expectedly small, $r$ remaining close to 3 .

Therefore, we predict the following general trend in semileptonic decays of unpolarized doubly heavy baryons: modes with a final state $\Sigma$-type $\left(\Sigma\right.$ or $\left.\Sigma^{*}\right)$ baryon should appear at a higher rate than those with a $\Lambda$-type baryon as a consequence of the (approximate) heavy quark spin symmetry in the preasymptotic mass region (moreover, recall that $\Lambda_{b c}, \Sigma_{b c} \rightarrow \Lambda_{c c} \ell \nu$ decays are forbidden and final states other than the ground-state $\Lambda_{c c}$ should be then considered in a more exhaustive analysis).

Future available data from the Tevatron [14] or LHC/SSC machines where doubly heavy baryons will be copiously produced [15] could permit to test our predictions for semileptonic decays and hence to determine the size of the corrections to these leading order evaluations. Furthermore, the possible consistency between our estimates in this work and those for the analogous decays of heavy-heavy mesons like charmonium, bottonium (which might be detected in a tau-charm factory, B-factory [5]) or $B_{c}$ mesons, should be useful to establish cross-checks of the heavy quark spin symmetry and the assumed factorization of the light and heavy components in the decay dynamics. Further comparisons could be extended to singly heavy mesons and baryons, offering altogether a general insight into the spectator behavior which is on the physical grounds of the phenomenological applications of HQET to the weak decays of hadrons. 


\section{References}

[1] M. Neubert, SLAC-PUB-6263 (1993), to appear in Physics Reports.

[2] M.J. Savage and M.B. Wise, Phys. Lett. B248 (1990) 177.

[3] E. Jenkins, M. Luke, A.V. Manohar and M.J. Savage, Nuc. Phys. B290 (1993) 463.

[4] R. Casalbuoni, A. Deandrea, N. Di Bartolomeo, R. Gatto and G. Nardulli, Phys. Lett. B302 (1993) 95; R. Casalbuoni et al, Phys. Lett. B309 (1993) 163.

[5] M.A. Sanchis, Phys. Lett, B312 (1993) 333; M.A. Sanchis-Lozano, FTUV 93-10 (1993).

[6] T. Ito, T. Morii and M. Tanimoto, Z. Phys. C - Particles and Fields 59 (1993) 57.

[7] M.J. White and M.J. Savage, Phys. Lett. B271 (1991) 410.

[8] J.G. Körner and M. Krämer, Z. Phys. C - Particles and Fields 55 (1992) 659.

[9] N. Isgur, D. Scora, B. Grinstein and M.B. Wise, Phys. Rev. D39 (1989) 799.

[10] M.J. Savage, private communication. An agreement on the incorrectness of the non-recoil limit of this transition shown in ref. [7] has been reached.

[11] M.B. Voloshin and M.A. Shifman, Sov. J. Nuc. Phys. 47 (1988) 511.

[12] Particle Data Group, Review of particle properties, Phys. Rev. D45 (1992).

[13] M. Suzuki, Nuc. Phys. B258 (1985) 553.

[14] A.F. Falk, M. Luke, M.J. Savage and M.B. Wise, SLAC-PUB-6226 (May 1993).

[15] A. Fridman and B. Margolis, CERN-TH 6878/93. 
